Check for updates

Cite this: RSC Adv., 2017, 7, 25265

\title{
Wettability tailoring of nanotube carpets: morphology-chemistry synergy for hydrophobic- hydrophilic cycling
}

\author{
Lvmeng He, Anil Karumuri and Sharmila M. Mukhopadhyay (D)*
}

Carpet-like arrays of carbon nanotubes (CNTs) on graphitic carbon materials have been investigated in order to understand all-carbon hierarchical structures for multifunctional surface-active devices. Pure CNT carpets are seen to be super-hydrophobic as long as they are well aligned. For future applications involving aqueous environments, the ability to tailor the surface wettability and switch it on demand can be very useful, and enable unprecedented devices related to microfluidics, catalysis and sensing/ detection systems. In this study, microwave plasma treatments were used to functionalize CNT carpets for a progressive increase in wettability so that they could eventually become super-hydrophilic. This change could be reversed by heating. Alternating between microwave plasma treatment and heating enabled repeated cycling of the CNT carpets between super-hydrophobic and super-hydrophilic states. This paper focuses on the influence of these two treatments on surface chemical states and multiscale morphology of CNT carpets, and their relation to wettability. It was shown by X-ray Photoelectron Spectroscopy (XPS) that oxygen-containing groups attached to surface carbon atoms are created during plasma treatment. These species desorb at temperatures of about $110{ }^{\circ} \mathrm{C}$. The strength of $\mathrm{C} 1 \mathrm{~s}$ and $\mathrm{O}$ is XPS signals from these radicals were seen to have direct correlation with water contact angles. In addition to surface chemistry, carpet morphology plays an important role in contact angle variations. Extreme surface roughness caused by high aspect-ratio of nanotubes would strongly accentuate both hydrophobic and hydrophilic behavior compared to flat surfaces. Classical geometric models of liquid droplets on uneven solids have been considered. Topological image analysis combined with intrinsic contact angle on flat graphene is used to predict the contact angle of these carpets, which matches well with experimental results. This analysis further explains why observed contact angles change if the vertical alignment of CNT is disturbed.

Received 6th March 2017 Accepted 1st May 2017

DOI: $10.1039 / c 7 r a 02745 a$

rsc.li/rsc-advances which have shown significant improvement in performance related to composite reinforcement, ${ }^{6}$ catalysis, ${ }^{7}$ thermal exchange, ${ }^{8}$ biological tissue scaffolding, ${ }^{9}$ charge storage, ${ }^{10}$ biosensing, ${ }^{11}$ and antibacterial activity for liquid purification. ${ }^{12,13}$

In further development of several of the above applications, infiltration of water or bio-fluids into the depths of CNT arrays will be an important factor for complete utilization of nanotube surfaces. For such applications, surface modification to control their hydrophilic/hydrophobic state is very important. Surface modification of isolated nanotubes are well studied and the large number of approaches discussed in the literature can be classified into two general functionalization approaches: wet chemistry and dry chemical processing.

In the wet chemistry approach, reactive chemicals such as acids are often used in solution to create defects on the surface. These may be are subsequently stabilized with carboxylic group $(-\mathrm{COOH})$ or hydroxyl $(-\mathrm{OH})$ groups,${ }^{14}$ or further modified by polymer coatings, esterification, and thiolation. Wet techniques need less complex facilities, but are often associated with chemical disposal issues. In the "dry" approaches, gases
Center for Nanoscale Multifunctional Materials, Department of Mechanical \& Materials Engineering, Wright State University, Dayton, OH 45435, USA. E-mail: smukhopa@wright.edu; Tel: +1-937-775-5092 
transport the active species for functionalizing the surface. Gases may be activated by external stimuli such as ultraviolet, electromagnetic field, UV/ozone and plasma. ${ }^{15}$ Plasma is becoming the activation method of choice in a large number of applications due to its high efficiency, good repeatability, and a wide range of precursor selection. ${ }^{16}$ In this study, we have focused on microwave plasma as the method of functionalizing carbon nanotubes, which has shown to be a versatile scalable approach that is easy to optimize as needed.

Surface wettability is an indicator of interfacial attraction or repulsion between the liquid phase and the solid phase. The interfacial energy per unit area $\left(\gamma_{\mathrm{sl}}\right)$ is determined by interatomic interactions between the chemical components of the solid and liquid. This, in turn, determines the inherent wettability which can be characterized by the static contact angle (CA) of a liquid droplet in contact with flat horizontal solid surface (Fig. 1A), as given by the classical Young's equation. ${ }^{17}$

$$
\gamma_{\mathrm{SG}}=\gamma_{\mathrm{SL}}+\gamma_{\mathrm{LG}} \cos \theta
$$

here, $\gamma_{\mathrm{SG}}$ solid-gas interfacial energy (sometimes referred to as surface tension); $\gamma_{\mathrm{SL}}$ is solid-liquid interfacial energy; $\gamma_{\mathrm{LG}}$ is liquid-gas interfacial energy; $\theta_{\mathrm{i}}$ is the inherent contact angle, assuming perfectly flat substrate. As the surface topology is altered, the overall "apparent" contact angle changes due to alterations in solid-liquid interfacial contact areas. Two common modifications to the Young's equation are proposed:

The Wenzel's model ${ }^{18}$ uses a roughness parameter to account for the fact that under a macroscopic liquid drop, the actual contact area between solid and liquid in a rough surface is higher than that in a flat surface (Fig. 1B). Contact angle is approximated to be:

$$
\cos \theta_{\mathrm{w}}=r \cos \theta_{\mathrm{i}}
$$

here, $r$ is ratio of actual surface of the rough solid in contact with liquid, and its topological area.

In the Cassie Baxter model, ${ }^{19}$ it is assumed that a fraction of solid surface in contact with liquid and the rest has trapped air pockets (Fig. 1C). It is expressed as:

$$
\cos \theta_{\mathrm{CB}}=f \cos \theta_{\mathrm{i}}+f-1
$$

here, $f$ is morphological factor and is defined as the area fraction of solid-liquid contact; $(1-f)$ is the area fraction of airliquid interface.

The most famous natural superhydrophobic surface is of course the lotus leaf, ${ }^{20}$ where water droplet can easily roll off and clean up dirt. In such surfaces a wax-like coating provides high intrinsic hydrophobicity, while its hierarchical morphology with two levels of roughness (microscale and nanoscale) provides the super-hydrophobic boost. Since then, several engineered surfaces have been shown to be superhydrophobic, using nanorod or nanoridge type surface features. Some of them were also shown to change behavior with chemical reactions. For instance, superhydrophobic $\mathrm{TiO}_{2}$ nanorod films on glass substrates are seen to become superhydrophobic when exposed to ultraviolet light, and reversed to superhydrophobic after storage in the dark, ${ }^{21}$ superhydrophobic $\mathrm{ZnO}$ nanorods films show contact angle decreasing after UV irradiation, ${ }^{22} \mathrm{SnO}_{2}$ nanowires on $\mathrm{SiO}_{x}$ become superhydrophobic after adding "brush-type" structure, ${ }^{23}$ $\mathrm{CuO} / \mathrm{Cu}(\mathrm{OH})_{2}$ nanowire surfaces lost their superhydrophobicity after a solution-immersion process. ${ }^{24}$ Similarly, boron nitride nanotubes that are superhydrophobic ${ }^{25,26}$ can be made to be hydrophilic by treating them in $\mathrm{N}_{2}-\mathrm{H}_{2}$ plasma.

For the case of carbon nanotube (CNT) carpets, there are few wettability studies reported, mostly grown on ideal electronic grade silicon or flat metallic substrates with pre-seeded catalysts. ${ }^{27-32}$ Since individual nanotubes are rolled-up graphene sheets, where atoms at edge and defect sites have significantly different chemical potential and reactivity compared to planer atoms, surface properties can depend significantly on processing technique, which determines graphitic stacking and defect densities. For instance, Wirth et al. reported a variable contact angle around $126^{\circ}$ on CNT carpets that decreases to $80^{\circ}$ after water exposure. ${ }^{27}$ Aria et al. reported that the as-grown CNT carpets were somewhat hydrophobic, which became superhydrophobic after vacuum pyrolysis treatment. ${ }^{15}$ Anderson et al. Reported a contact angle of $154^{\circ}$ on as-grown CNT carpet. Li et al. stated contact angle of $150^{\circ}$ on as-grown CNT. ${ }^{28}$ Ramos et al. reported that $\mathrm{CNT}$ grown on Ti substrates treated with oxygen pulsed DC plasma can be hydrophilic, whereas $\mathrm{CO}_{2}$ laser treatment can make it super-hydrophobic with contact angle up to $160^{\circ} .^{29,30}$ Similarly Kafi et al. ${ }^{31}$ and Chen et al. ${ }^{32}$ have used $\mathrm{N}_{2}-$ $\mathrm{H}_{2}$ and steam plasmas respectively to make CNT hydrophilic.

Our team has focused on investigation of CNT carpets grown on graphitic substrates using a two-step scalable technique: ${ }^{5}$ deposition of oxide buffer layer using microwave plasma, followed by CNT attachment using floating catalyst chemical vapor deposition (FC-CVD). This technique has the distinctive advantage that it can be applied to porous and uneven substrates such as carbon fabric and aerospace foams suitable for strong, lightweight and ecofriendly devices. The long-term objective of this effort is to understand and develop all-carbon hierarchical materials for a variety of applications..$^{5-13}$
A

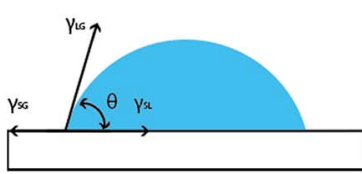

B

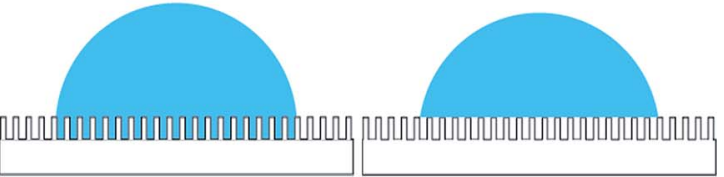

Fig. 1 Schematic models that lead to Young's equation (A), Wenzel's approximation (B) and Cassie Baxter approximation (C). 
However, it must be noted that since CNT arrays are known to depend on both substrate characteristics and nanocatalyst distribution, the carpet grown in these materials are expected to be different from those grown by earlier reported techniques. In particular, earlier studies involving CNT arrays were grown on epitaxial oxides on silicon or metal, which tend to be crystalline and smooth. On the other hand, our samples are grown on amorphous silica formed in plasma phase and known to have uneven cauliflower-type morphology. ${ }^{5}$ Additionally, the earlier reported studies mostly involve pre-deposited transition metal catalysts to provide uniform nucleation sites on which nanotubes grow for a fixed time. In contrast, we have used the more scalable floating catalyst (FC-CVD) technique where iron catalyst particles (nucleation sites) are continuously being deposited and new nanotubes nucleating throughout the CVD growth. This implies less uniform carpets compared to those reported earlier. The structural and compositional details of this new family of CNT have been investigated at length and discussed in earlier studies, as briefly explained in section 4 .

Some aspects of surface wettability in these materials, and their influence on fluid-based applications have also been discussed more recently. ${ }^{33,34}$ It was shown that CNT carpets, as fabricated, are free from detectable defects and impurities, and tend to be super-hydrophobic. They can be made permanently hydrophilic with liquid phase silica coatings and temporarily hydrophilic through microwave plasma treatments. These modifications are seen to have significant influence on diverse functionalities of CNT carpets such as nanoparticle attachment, water purification rates and tissue proliferation. This paper takes a focused, in-depth, look into the reversible water permeation changes created by dry plasma treatment. Vertically aligned CNT carpets have been grown on model flat graphite surfaces for detailed analyses. Observed contact angles have been correlated with surface electronic states analyzed by X-ray Photoelectron Spectroscopy (XPS) and with both microscale and nanoscale morphologies analyzed by Field Emission Scanning Electron Microscopy (FE-SEM). Variation of water contact angle have been investigated as a function of surface treatment times, and observed values compared with those predicted by uneven solid wettability models proposed in the literature. This study provides deeper insights into the possible ranges of surface wettability possible for CNT carpets, and how that can be tailored for advanced devices in future.

\section{Materials and methods}

\subsection{Synthesis}

For this study, commercial pressed graphite pieces were polished with low-grid and high-grid sand papers subsequently. Then, the graphite were sonicated in water and dried in ambient air at $110{ }^{\circ} \mathrm{C}$. A nano-layer of amorphous silica were deposited on the graphite pieces using microwave plasma enhanced chemical vapor (M-PECVD) prior to CNT growth. CNT were fabricated using floating catalyst chemical vapor deposition (CVD) in a horizontally tubular reactor. Xylene $\left(\mathrm{C}_{6} \mathrm{H}_{4} \mathrm{C}_{2} \mathrm{H}_{6}\right.$, PTI Process Chemicals) as carbon source and ferrocene $\left(\mathrm{Fe}\left(\mathrm{C}_{5} \mathrm{H}_{5}\right)_{2} 99 \%\right.$, Alfa-Aesar Ltd) as the catalyst source were introduced in the atmosphere of $\mathrm{Ar}$ and $\mathrm{H}_{2}$. Details are provided in earlier publications. ${ }^{5}$

\subsection{Surface treatments of CNT carpets}

Plasma treatment for increasing hydrophilic behavior - CNT carpets were treated using microwave oxygen plasma. CNT attached to graphite substrate was placed on the microwavedriven electrode chamber at room temperature, and MW power and time were varied.

On the other hand, thermal treatment was used to reverse hydrophilicity. In this method, heat treatment was carried out in ambient air at $110^{\circ} \mathrm{C}$.

\section{Characterization}

\subsection{Microstructure characterization}

Field emission scanning electron microscope (JEOL 7401FESEM) was used for the morphology of CNT surface.

\subsection{Surface chemistry characterization}

X-ray photoelectron spectroscopy (Krato Axis Ultra) with monochromatic Al-K $\alpha$ source $(1486.6 \mathrm{eV})$ was used to obtain the elemental information of CNT and their chemical states.

\subsection{Surface wettability measurement}

Contact angles were measured with in-lab built goniometer. All measurements were conducted in air at standard STP conditions. The assembly of this goniometer has been published previously. ${ }^{5}$ Each data point were averaged from five different positions.

\section{Results and discussion}

\subsection{Morphology of CNT carpets}

Earlier studies ${ }^{35}$ have described the structure, quality and density of individual nanotubes grown by this method. Extensive FESEM and TEM analysis had been used to estimate the average outer diameter, inner diameter, and number of walls to be $18 \mathrm{~nm}, 8 \mathrm{~nm}$ and 15 respectively. Moreover, it had been determined that the aerial density of nanotubes (average number of CNT strands per unit area of substrate) was about 1.7 $\times 10^{2} \mu \mathrm{m}^{-2}$ (or $\left.1.7 \times 10^{14} \mathrm{~m}^{-2}\right)$.

The quality of CNT, in terms of amorphous $v s$. graphitized carbon, is usually estimated from Raman spectra. Raman analysis of these materials performed earlier ${ }^{36}$ have shown that there is no peak in the radial breathing zone $\left(\sim 200 \mathrm{~cm}^{-1}\right)$, implying the absence of single walled CNTs. For the multiwalled CNT, the graphitic ( $\mathrm{G}$ band) to defective/amorphous (D-band) intensity ratio $\left(I_{\mathrm{G}} / I_{\mathrm{D}}\right)$ is used as an indicator of CNT quality. A higher $\left(I_{\mathrm{G}} / I_{\mathrm{D}}\right)$ implies better graphitization whereas lower ratio indicates increase in defects and amorphous carbon. Most studies on multi-walled CNT arrays reported in the literature show noticeable amount of defective carbon, with $\left(I_{\mathrm{G}} / I_{\mathrm{D}}\right)$ ratio in the range of 0.4 to 1.4 . Our samples ${ }^{36}$ have shown the $I_{\mathrm{G}} /$ $I_{\mathrm{D}}$ ratio to be consistently higher than 1 (mostly around 1.2) in 
as-synthesized as well as surface-treated samples, which indicates relatively high quality MWCNT throughout this study.

For this paper, we have focused on the topographic morphology of the CNT carpet grown on graphite surface. Surface morphology of CNT grown graphite surface can be seen in Fig. 2. Fig. 2A and B show the side view of the CNT array in low and high magnifications respectively. The inset in Fig. 2B shows a very high magnification image captured in transmission mode to indicate details of individual nanotubes. It can be seen that the CNT arrays grow as parallel bundles perpendicular to the substrate, and the top of the carpet has a microscale roughness with valleys and hills. These bundles are composed of entangled CNTs as seen from Fig. 2B. This hierarchical morphology therefore consists of two levels of roughness, the microscale variation of bundle height, and the nanoscale cylindrical feature of the individual nanotubes. Based on microstructural images in Fig. 2A and B, a schematic
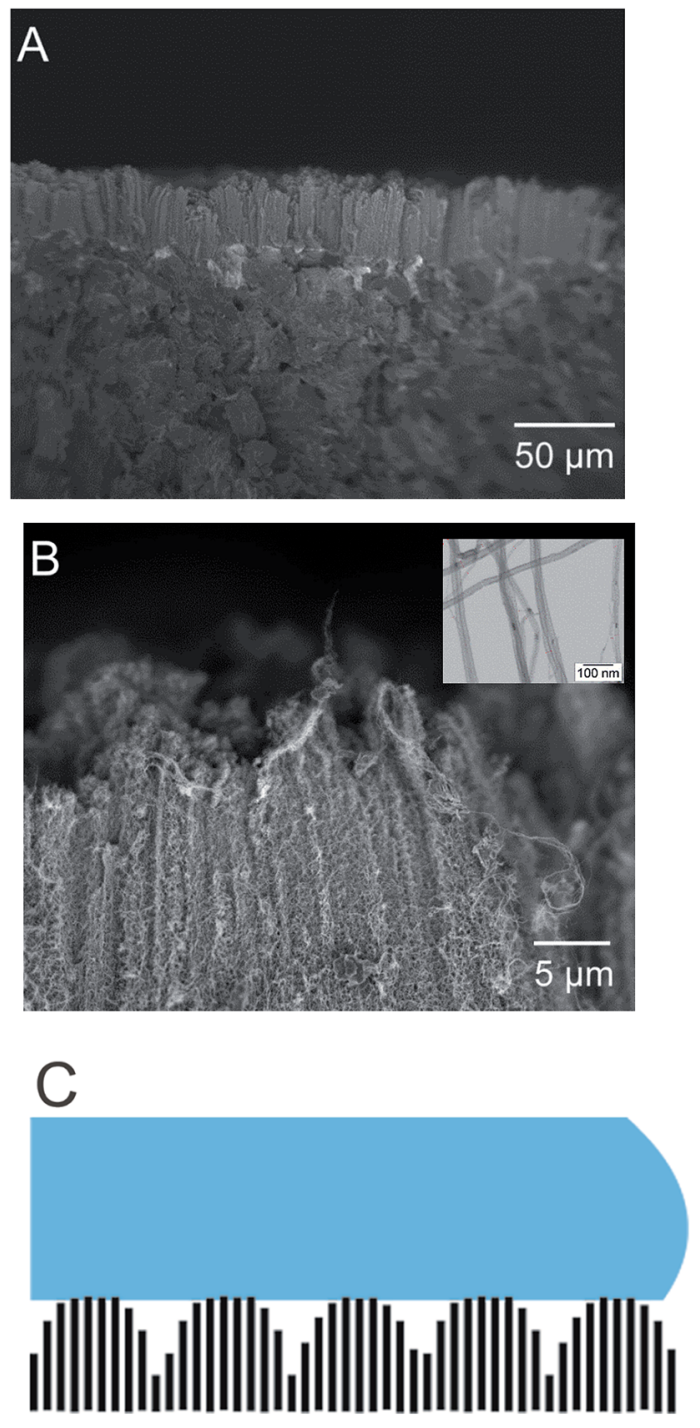

Fig. 2 Cross sectional side view of as-grown CNT carpets (A), high resolution SEM image of the carpet $(B)$, schematic view of the macro and microscale roughness of the carpets (C). of the carpet topology has been drawn in Fig. 2C for topographical roughness analysis. These topological roughness factors are estimated from SEM images and nanotube parameters, and used to interpret the observed wettability variations, as discussed later.

\subsection{Influence of surface treatments on morphology}

Fig. 3 shows the top (aerial) view of the CNT carpets before and after surface treatments. Figures on the left show low magnification and those on the right show high magnification images of untreated CNT (u-CNT) carpet (Fig. 3A and D) in comparison with plasma treated CNT (p-CNT) surface (Fig. 3B and E) and heat treated (h-CNT) after plasma treatment (Fig. 3C and F). It can be seen that the morphology is unchanged with these treatments. Functionalization of CNT with plasma involves attachment of functional groups and alterations in surface atomic bonds. However, these atomic level changes clearly donot have morphological impact at micro and nano scales detectable in high resolution SEM images.

\subsection{Influence of surface treatments on CNT carpet wettability}

Contact angle of a water droplet is used as the measure of wettability in this study. As-grown pure CNT carpets were found to be super-hydrophobic, meaning the contact angle is larger than $150^{\circ}$. Subsequently, when exposed for short times to low-
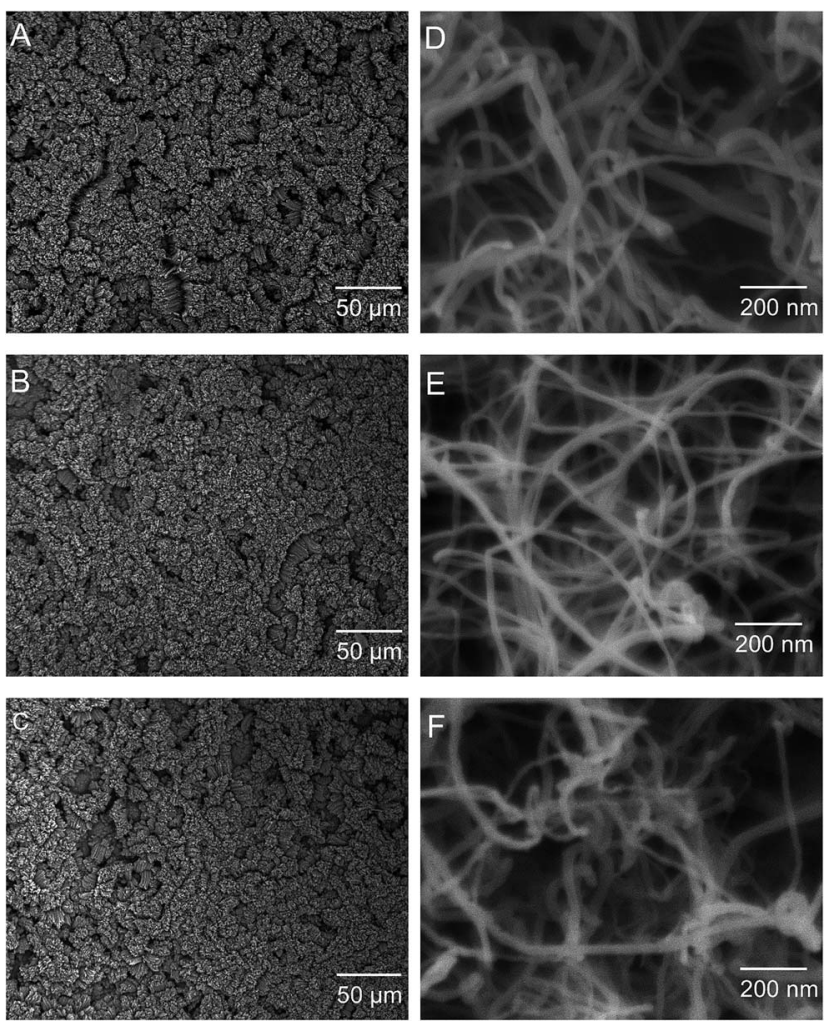

Fig. 3 Top down images of CNT carpets: as grown CNT carpets (A \& $D)$; plasma oxygen treated with $115 \mathrm{~W}$ for 10 seconds (B \& E); and heat treatment after plasma at $110^{\circ} \mathrm{C}$ for $3 \mathrm{~h} \mathrm{(C} \mathrm{\&} \mathrm{F).}$ 
power microwave induced oxygen plasma, the contact angle is seen to decrease. Fig. 4 shows images of water drops on the CNT carpet as a function of plasma duration. The insets show pictures of a water droplet with contact angle larger than $160^{\circ}$ before plasma to less than $20^{\circ}$ after $6 \mathrm{~s}$ of exposure. It must be noted that in this type of three dimensional hierarchical surface architecture, contact angle below 20 degree cannot be accurately measured due to the liquid slowly seeping into the carpet. What is clear is that initially, the contact angle decreases almost linearly with plasma exposure, then saturates to a very low value (clearly hydrophilic behavior) in about 6 seconds.

This effect of increased wettability is seen to be easily reversed if the material is gently heated $\left(110{ }^{\circ} \mathrm{C}\right.$ in this case) in air. To study the influence of heat treatment on plasma oxygen exposed CNT on contact angle, CNT carpets exposed to different plasma times ( $4 \mathrm{~s}$ and $10 \mathrm{~s}$ ) were heated for different times and their contact angles measured. Fig. 5 shows the effect of heating on two different plasma treatment times having different levels of hydrophilicity. In both cases, the contact angle of the CNT carpet increased with heat treatment time. After about 3 hours of heat treatment in air, super-hydrophobic state of pure untreated CNT was restored. This implies that the functional species attached on the CNT surface, responsible for hydrophilic behavior, has relatively weak binding with the nanotube surface, and can be removed by simple air-heating. It must be noted that similar effect of functional group removal (and wettability reversal) was also observed when the samples were stored in desiccator environment for prolonged periods (weeks). As plasma treatments are gaining popularity in device fabrication, this observation is being reported by multiple investigators, ${ }^{37-40}$ who have attached different types of plasmagenerated functional groups (especially polar groups) on polymeric and carbon substrates. Earlier studies indicate that surface chemistry and related property alterations caused by plasma treatments can degrade with time. Some authors refer to this as "aging" of the surface, ${ }^{38}$ whose rate is expected to increase with temperature. Several possible mechanisms have been suggested, ranging from reorientation or diffusion of polar surface groups to reaction or bond-degradation in air. For our

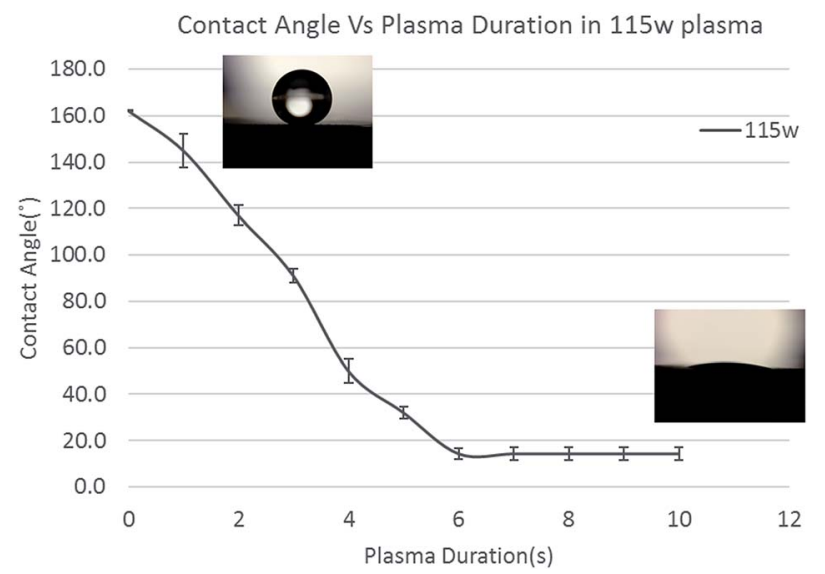

Fig. 4 Influence of oxygen plasma processing conditions on contact angle.

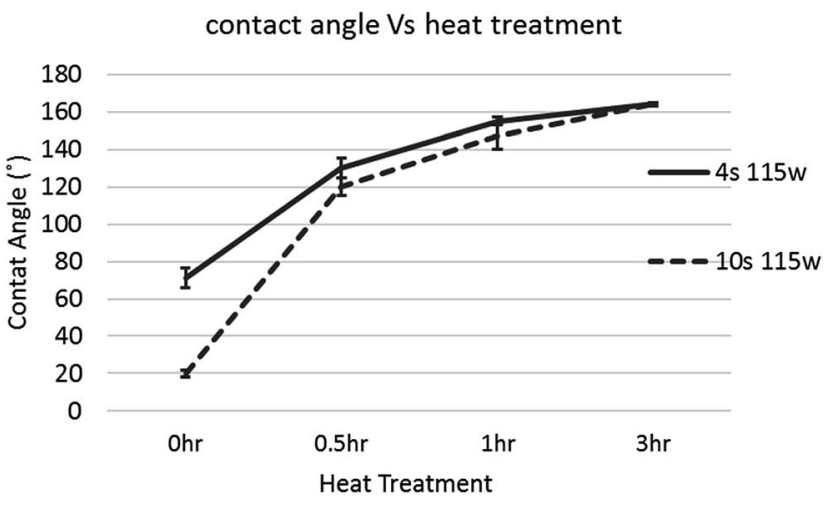

Fig. 5 Influence of reduction process parameters, heat treatment on oxygen plasma treated CNT.

samples, XPS data clearly indicates loss of the $\mathrm{O} / \mathrm{OH}$ species attached to carbon, so the former explanation is not valid. Moreover, if the functional groups were retained on the surface in some other orientation or agglomerated form, repeatable cycling between super-hydrophobic-super-hydrophilic states would not have been possible. Therefore, the most likely explanation is that the functional species deposited by plasma are detached from the CNT surface with prolonged storage, which can be accelerated by heat. These groups can be reattached with subsequent plasma treatments, and multiple cycles of attachment-detachment are possible. The fact that rapid detachment can occur at relatively low $\left(110^{\circ} \mathrm{C}\right)$ temperature implies that the bond dissociation energy of CNT-functional group is low, maybe close to that of water vapor. In-depth computational thermodynamic investigations relating these observations with specific plasma components in this process and interatomic bond energies will be very useful and are being initiated. These will be reported in future.

From application perspective, this study underscores the limited durability and "shelf life" of low binding energy functional groups attached by plasma on CNT carpets. This study clearly indicates that functional groups that are created on the CNT surface by short duration microwave oxygen plasma are very effective in imparting temporary hydrophilic behavior, which can be rapidly reversed by heat treatment as needed, and repeated cycling is possible.

\subsection{Influence of wettability on capillary action and CNT morphology}

These experiments also reveal an important aspect of CNT carpets in how they may interact with static water droplets. Whereas short duration plasma treatments and air annealing did not show any change in carpet morphology, the static contact angle (CA) test on plasma-treated hydrophilic surfaces created localized change in carpet morphology at the water droplet site. Visible "spots" on the carpet could be seen where the water droplet was introduced and then allowed to evaporate. Microscopic analysis showed crater-like regions at such sites caused by collapse of CNT carpets. Fig. 6 shows an image from the edge of such a crater, and schematic of CNT topology is shown in the 


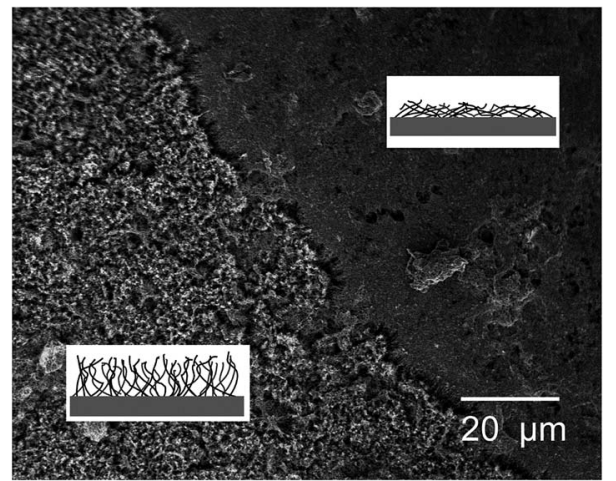

Fig. 6 Influence of capillary driven drying on CNT carpet morphology.

inserts. This type of collapse in surface nanotube arrays has been reported by earlier investigators ${ }^{41}$ and attributed to capillary forces during fluid infiltration and evaporation. The fiber aggregation model predicted by Py et al. ${ }^{42}$ predicts that parallel flexible fibers (nanotubes in this case) tend to aggregate when the free ends are immersed in a highly wetting liquid. This type of CNT aggregation causes aligned arrays to become densified bundles or strands, and have potential applications in novel conducting interconnects, microfluidic devices, cell seeding, and pressure sensitive adhesives. ${ }^{43,44}$

In context of the current study, nanotubes in their hydrophilic state that are immersed in the water droplet stick together and are bent towards the center of the water droplet by capillary forces. After the water evaporates, they retain this distorted morphology due to van der Waals forces. This explains the appearance of "collapsed spots" in top view. The direction and morphology of aggregated (collapsed) CNT strands may depend on CNT carpet height, size of water drop, and kinetics of water-vapor transport during evaporation, and will be of interest for future investigations related to microfluidic device channels. For this study, it is shown in the next section that the collapsed regions show a different pattern of hydrophobic/ hydrophilic cycling compared to un-collapsed CNT carpets that were not subjected to static water drops.

\subsection{Hydrophobic-hydrophilic cycling of CNT nano-carpets}

Since the CNT carpets could be made hydrophilic by plasma treatment and returned to hydrophobic state by mild heating, the possibility of repeated cycling between the two states was tested by subjecting them to multiple cycles. Plasma treatment times were varied to give different levels of hydrophilic behavior. Heat treatments were conducted at $110{ }^{\circ} \mathrm{C}$ for 1 hour. It was clear that the hydrophobic-hydrophilic reversal could be repeated multiple times, indicating that it is possible to switch between different states as needed. A noteworthy observation is that complete back and forth switching between superhydrophobic (contact angle $160^{\circ}$ ) and super-hydrophilic (unstable contact angle $20^{\circ}$ or below) depends on the retention of CNT carpet alignment.

Fig. 7 shows two types of cyclic behavior seen on these carpets, one when each contact angle measurement was taken on pristine areas (Fig. 7A) that had not been subject to water droplet test, and the other where the contact angle measurement was taken on a region of the carpet that had collapsed (Fig. 7B) earlier due to water droplet tests. In the case of pristine upright CNT, plasma treatment caused strong reduction in CA that could be completely reversed by air heating. Hence multiple cycles were possible with no detectable change in behavior. However, in regions where the CNT had collapsed due to water test, complete reversal of hydrophobicity was not observed and a gradual decrease in hydrophobicity could be seen with each cycle of hydrophilic/hydrophilic treatment. This difference can be explained in terms of change in surface morphology leading to change in effective contact area at the CNT-water interface, as discussed in the last section (4.7).

\subsection{Relation between surface treatments and chemical states}

The surface chemical states of the CNT carpets were analyzed using X-ray photoelectron spectroscopy (XPS). Three types of CNT carpets are analyzed: as-grown untreated CNT carpets (uCNT), CNT carpets that have been treated with oxygen plasma (p-CNT), and CNT carpets treated with plasma, followed by heat treatment (h-CNT). High resolution peaks (with scanning steps at $0.1 \mathrm{eV}$ ) were taken of the $\mathrm{C} 1 \mathrm{~s}$ and $\mathrm{O} 1 \mathrm{~s}$ photoelectron peaks, and mathematical peak fitting with possible atomic bonding states performed. This type of deconvolution exercise can help provide semi-quantitative estimates of each type of bonding state in a given atomic species (carbon and oxygen in this case) that exists in the near-surface region of the solid.

The $\mathrm{C}$ 1s peak shapes for all three samples, u-CNT, p-CNT, and h-CNT are shown in Fig. 8A. As shown in Fig. $8 \mathrm{~B},{ }^{33} \mathrm{C}$ 1s peak could be deconvoluted into the main $\mathrm{C} 1 \mathrm{~s}$ peak at $284.6 \mathrm{eV}$ and a Pi-Pi peak at $291 \pm 0.5 \mathrm{eV}$ respectively. ${ }^{45}$ In case of p-CNT, besides C $1 \mathrm{~s}$ and Pi-Pi peaks, a C-O/OH component was seen at $287 \mathrm{eV}$, which confirms the presence of $\mathrm{C}-\mathrm{O} / \mathrm{OH}$ after functionalization with oxygen plasma. Upon heat treatment, the $\mathrm{C}-\mathrm{O} / \mathrm{OH}$ component on h-CNT was reduced to 0.9 at $\%$ from initial 3 at $\%$. The results indicate that the component of $\mathrm{C}-\mathrm{O} / \mathrm{OH}$ can be increased by oxygen plasma and reduced by heat treatment. A systematic correlation was made between the intensity of the $\mathrm{C}-\mathrm{O} / \mathrm{OH}$ component of the $\mathrm{C} 1 \mathrm{~s}$ peak and observed water contact angle. This is shown in Fig. $8 \mathrm{C}$ and clearly indicates that the contact angle decreases as the hydroxyl component $(\mathrm{C}-\mathrm{O} / \mathrm{OH})$ increases.

In addition to $\mathrm{C} 1 \mathrm{~s}$ peak, the $\mathrm{O}$ 1s peak of CNT carpets were also analyzed. The overall survey scan of the $\mathrm{O} 1 \mathrm{~s}$ region from each sample have been are superimposed to show relative oxygen concentrations in Fig. 9A. Individual components were also deconvoluted into $\mathrm{O}-\mathrm{C}$ at $533.1 \mathrm{eV}$ and $\mathrm{OH} / \mathrm{O}-\mathrm{C}$ at $531.9 \mathrm{eV}$. These have been shown in Fig. 9B-D. It is clear that the total intensity of $\mathrm{O} 1 \mathrm{~s}$ peak was increased after oxygen plasma (pCNT) and reduced after heat treatment. In addition, the peak was shifted from $533 \mathrm{eV}$ to $532 \mathrm{eV}$ after plasma and shifted back to $533 \mathrm{eV}$ after following heat treatment. The $\mathrm{OH} / \mathrm{O}-\mathrm{C}$ component at $531.9 \mathrm{eV}$ is attributed to the weakly bonded plasmainduced oxygen group attached to the carbon nanotube. ${ }^{46} \mathrm{It}$ can be seen that the $\mathrm{C}-\mathrm{O} / \mathrm{OH}$ fraction increased after plasma treatment and decreased following heat treatment. Fig. 9E 

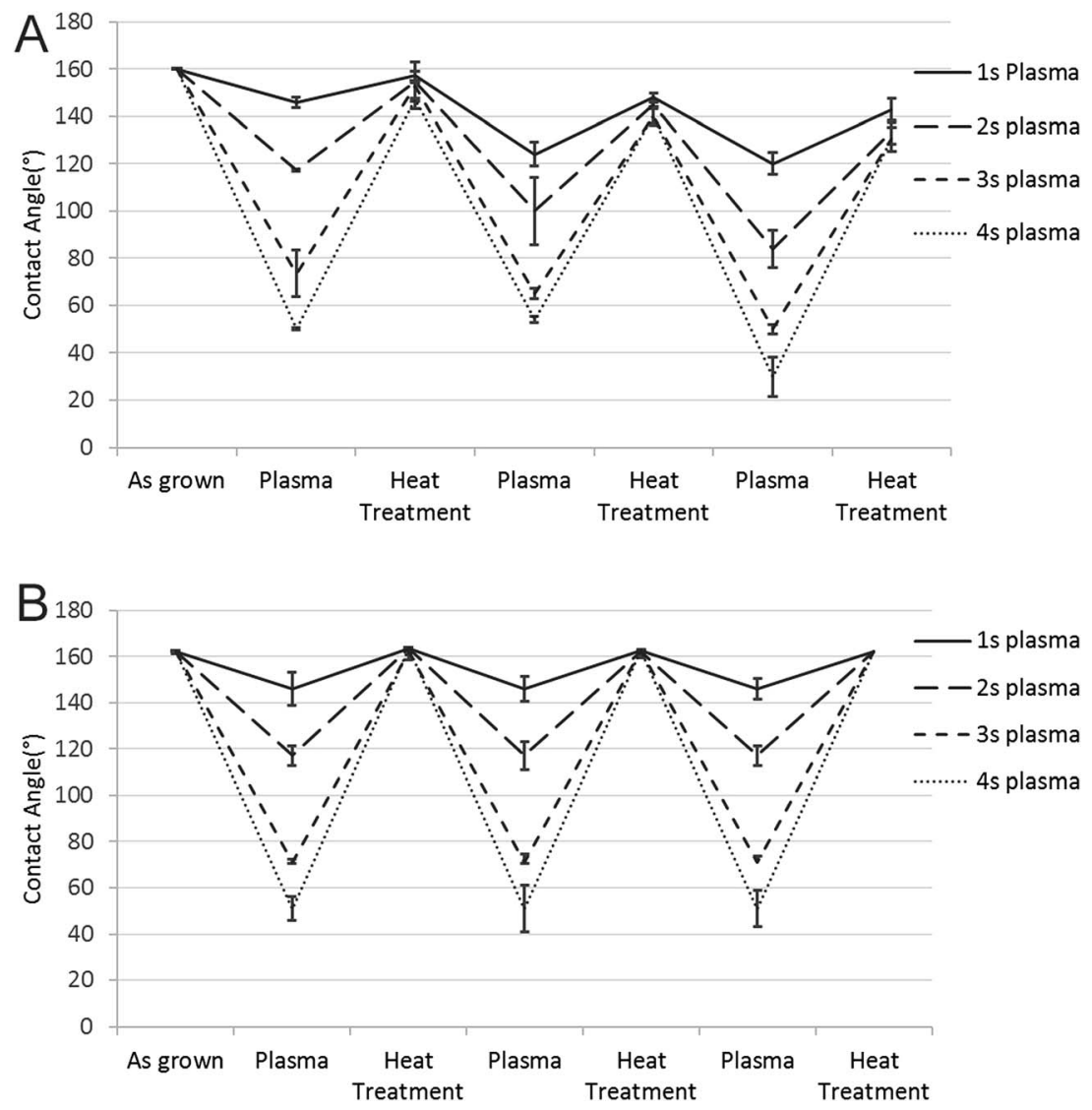

Fig. 7 Cycling of water droplet on collapsed (A) and pristine CNT (B) carpets.
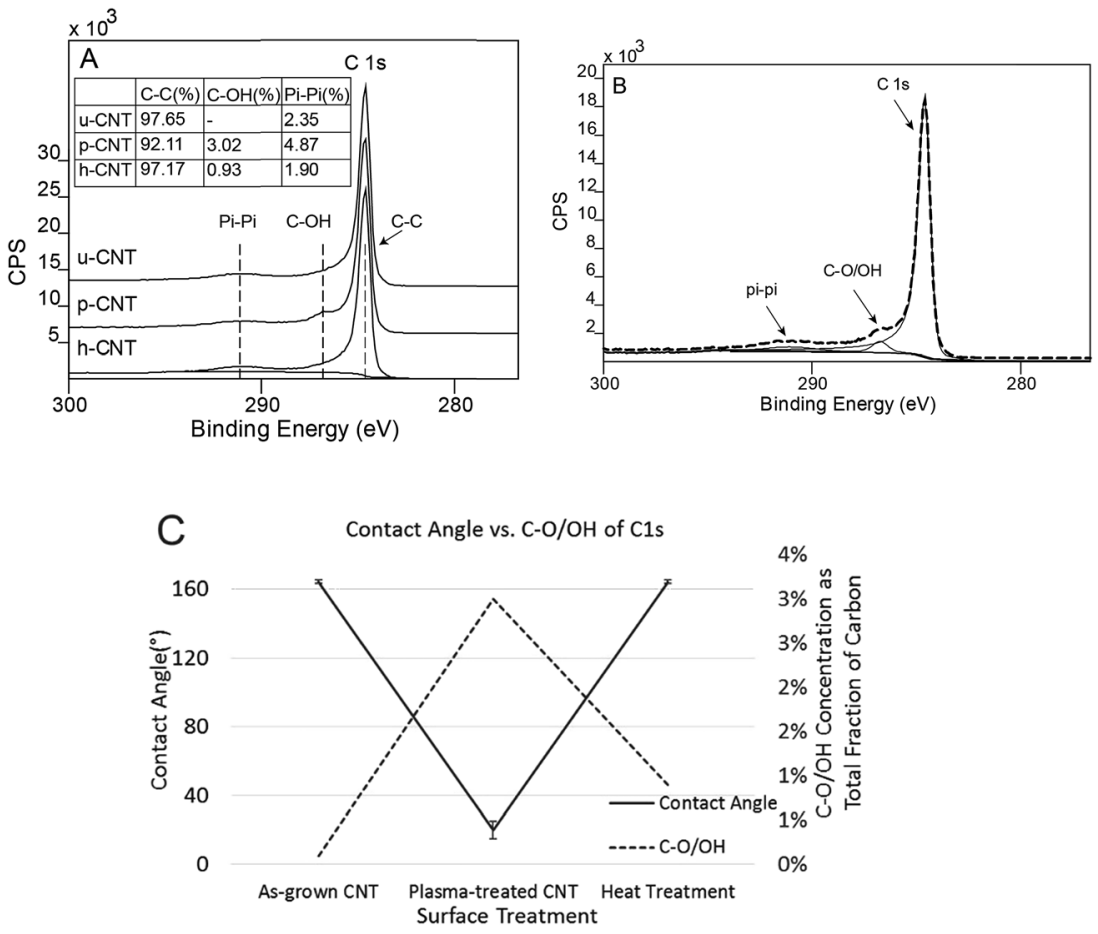

Fig. $8 \mathrm{C}$ 1s photoelectron peak collected on u-CNT, p-CNT, h-CNT (A); deconvolution of components of C 1s peak (B); influence of contact angle on fraction concentration of $\mathrm{OH}-\mathrm{C}$ groups (C). 

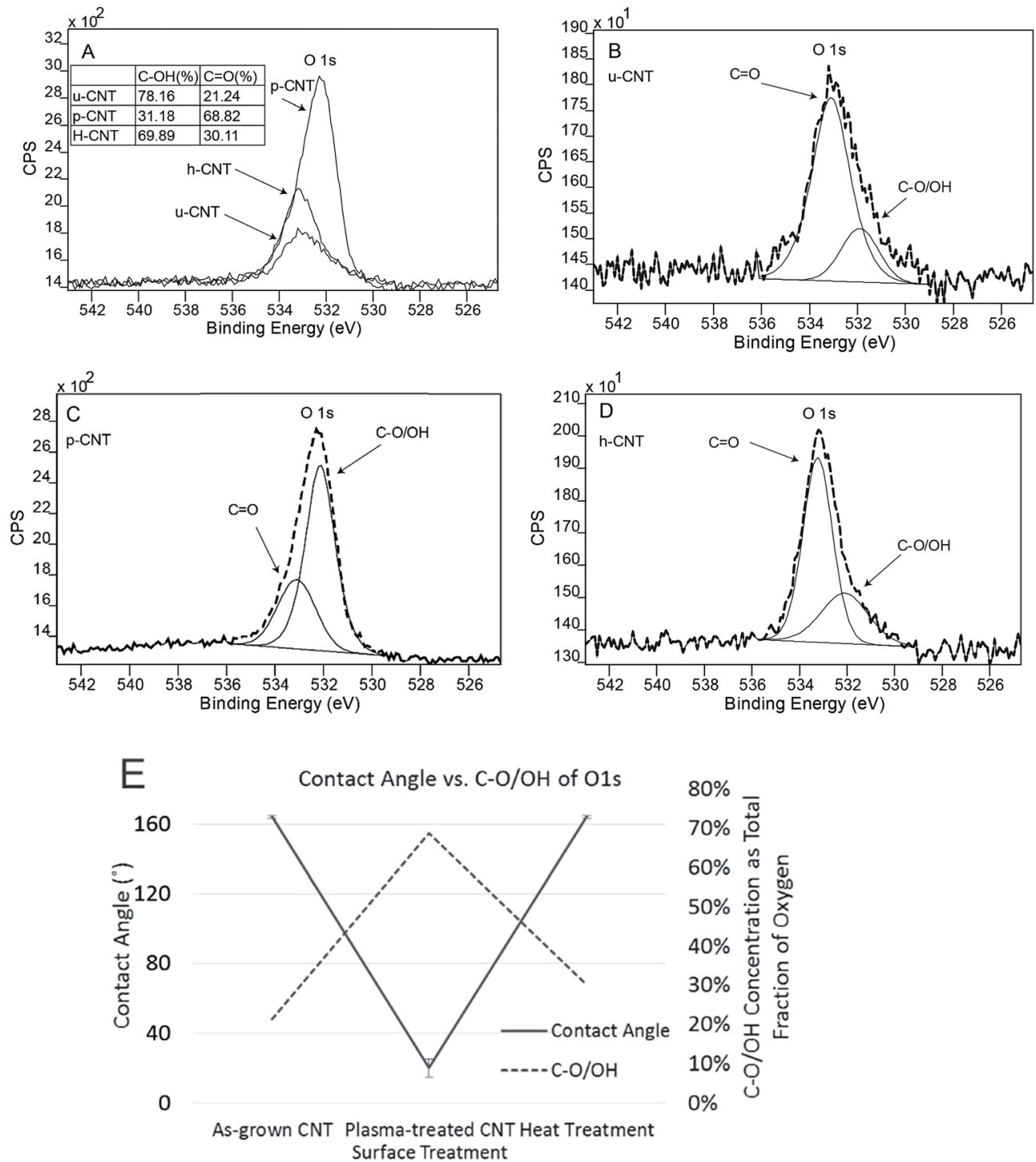

Fig. $9 \mathrm{O}$ 1s photoelectron peaks collected on u-CNT, p-CNT, h-CNT (A); Each individual O 1s peak is deconvoluted to show the component peaks: $u$-cnt (B), p-cnt (C), h-cnt (D) respectively; influence of contact angle on fraction concentration of $\mathrm{OH}-\mathrm{C}$ groups (E).

shows that the contact angle variation of the $\mathrm{C}-\mathrm{O} / \mathrm{OH}$ component in the oxygen peak correlated qualitatively with the contact angle variation in the same way that the $\mathrm{C}-\mathrm{O} / \mathrm{OH}$ component in the carbon photoelectron peak shown in Fig. 8C. This clearly proves that the $\mathrm{C}-\mathrm{O} / \mathrm{OH}$ component is the one introduced by oxygen plasma and reduced by air annealing and that this group is directly responsible for the wettability change. The fact that they are removed by heating at $110^{\circ} \mathrm{C}$ implies that the binding energy between CNT and these molecular groups is relatively low, useful for applications that may require rapid switching between two states.

\subsection{Contact angle model for CNT carpet: mathematical prediction vs. experimental observation}

It is important to see if and under what conditions, existing mathematical models of solid wettability by a liquid can be applicable to CNT carpets. ${ }^{47}$ As discussed in section 1 , there are two commonly accepted modifications to the Young's equation of wetting when the solid has uneven surface morphology. They are the Wenzel and Cassie Baxter models.

Since the Wenzel equation assumes that the liquid is fully in contact with the solid, which implies complete permeation into CNT carpets, it will only be applicable for the hydrophilic surface state. This model simply adds a roughness factor $(r)$ to account for increased surface area of contact as shown in eqn (2).

For compact solids, roughness $r$ (or some authors prefer the root mean square roughness, RMS) are statistical numbers depending upon surface bumps. Roughness $r$ in this model indicates the ratio of actual solid-liquid interface area to the topological area of ideally flat substrate. It must be noted that for these types of hierarchical surfaces ${ }^{47}$ having arrays of upright nanoscale features with aspect ratios (length/diameter) of the order of $1000 / 1,(r)$ can be close to the aspect ratio, 
depending on the level of topological entanglement. In other words, the value of $r$ can be extremely high, which will cause excessive amplification of both hydrophobic and hydrophilic effects. Therefore, even a small extent of hydrophilic behavior (when $\theta$ is less than 90 and $\cos \theta$ is positive) will lead to significant increase in effective wettability and infiltration/ spreading of water drop on surface. On the other hand, if such materials are even mildly hydrophobic (when $\theta$ is greater than 90 and $\cos \theta$ is negative), water will be repelled from the surface, resulting in air gaps within the nanotube features, making Wenzel model inadequate and Cassie Baxter model more appropriate as discussed below.

The Cassie-Baxter model assumes that the liquid makes only partial contact with the solid, with air pockets trapped within the interfacial features. This is therefore suitable for insufficient wetting or hydrophobic solids and is shown in eqn (3). In this model, $\theta_{\mathrm{CB}}$, the observed Cassie Baxter contact angle depends on the inherent contact angle, $\theta_{\mathrm{i}}$ and $f$, the morphological factor defined as the area fraction of solid-liquid area. $(1-f)$ is the area fraction of air-liquid area.

Since the CNT surface can be assumed to be rolled up graphitic carbon sheets, the inherent contact angle for graphite should be applicable for $\theta_{\mathrm{i}}$. Several in-house tests were performed and literature reports compiled to estimate the inherent contact angle of water on flat graphite substrates, and wide variations of contact angle values could be seen $\left(89-115^{\circ}\right)$. It was finally determined that reported values for the purest form of graphitic surface (graphene sheets) should be used as best estimates of $\theta_{\mathrm{i}}$ on pure carbon nanotubes. Contact angle of pristine graphene surfaces reported in the literature, obtained by both first principle simulation and careful testing on graphene were reported between $95-107^{\circ} \mathbf{4 8 , 4 9}$ and this is the range of $\theta_{\mathrm{i}}$ used for this study. The morphological factor is discussed as follows.

Area fraction in contact $(f)$. The morphological parameter in Cassie-Baxter model is represented by the ratio of solidliquid contact area between the water droplet and upper tip of the CNT carpet. A qualitative estimate can be made based on microstructural image analysis. As shown in Fig. 1, roughness at both microscale and nanoscale exist at the tip, and should be considered to estimate the contact angle. The CNT carpet topology consists of micro bumps, and each bump consists of individual nanotube coils and tips. Microscale contact fraction $\left(f_{\mathrm{m}}\right)$ can be estimated by detailed image analysis of the carpets using low magnification and microscale resolution, whereas nanoscale contact fraction within a bump $\left(f_{\mathrm{n}}\right)$ is estimated from topological image analysis of higher magnification (nanoscale resolution) images where profile of individual nanotubes at different depths can be distinguished. The overall fraction $f$ will be the product of $f_{\mathrm{m}}$ and $f_{\mathrm{n}}$.

Image contrast approach has been used to analyze the topological images, similar to technique reported by earlier authors $^{50}$ who quantitatively estimated the area fraction of boron nitride carpets. It is assumed that pixels in different brightness in the SEM images indicate different heights of CNT carpets. Two types of pixels are considered: those above
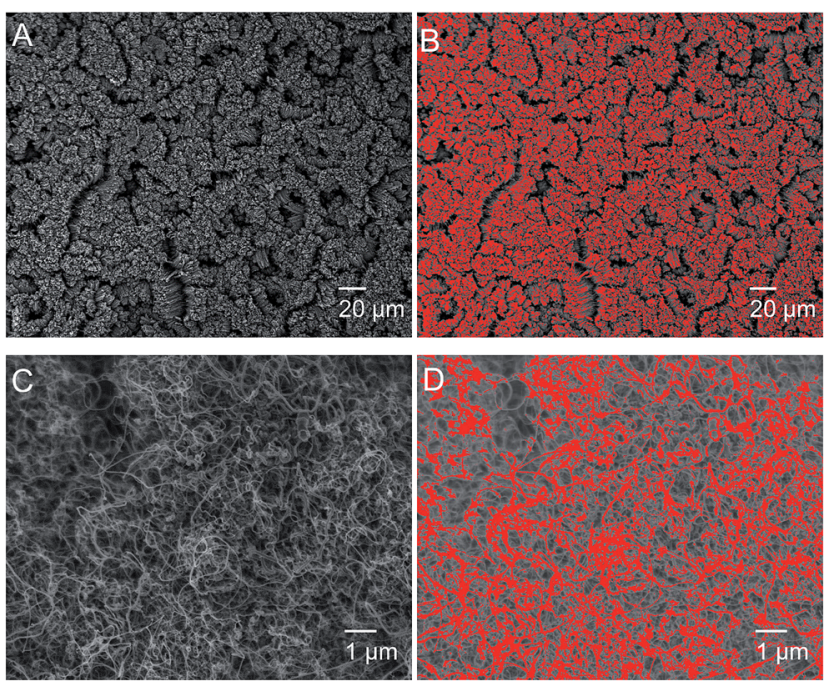

Fig. 10 Microstructures of CNT surfaces before (A) and after (B) selecting bright pixels at low magnification; higher resolution images to determine nanostructure before (C) and (D) after selecting bright pixels at high magnification.

a certain brightness are considered as top surface that contact the water, and pixels below this brightness are considered as non-contacting pixels. Although the selection of cut-off brightness does depend on researchers' judgment, the random errors could be minimized by averaging similar measurements from a large number of images. The image processing software ImageJ was used to distinguish the contacting and non-contacting regions. Sample images at two magnifications as shown in Fig. 10A and C. Fig. 10B shows contacting regions at lower magnification images to estimate the microscale bumps, and Fig. 10D shows bright pixels (top of surface) at higher magnification images to identify contact fraction at the scale of individual nanotubes. Such estimates averaged over a large number of images indicate a microscale fraction of $f_{\mathrm{m}}=0.33$ and nanoscale fraction of $f_{\mathrm{n}}=0.14$. The overall area fractions is therefore the product of the two, which is 0.0462 . By applying the $f$ value to the inherent contact angle range $\left(95-107^{\circ}\right)$ in Cassie-Baxter equation, one can obtain the estimated contact angle of $163.3-165.3^{\circ}$, which agrees with our experimentally obtained value of about $165^{\circ}$. This analysis indicates that even though graphene surfaces is expected to be slightly hydrophobic, the unique morphology of vertically aligned CNT carpets make them super-hydrophobic.

This model also explains why the contact angle of collapsed carpets (as seen in Fig. 6) was observed to be lower than that of original upright and vertical carpets. Collapsed carpets will offer higher fraction of topology in contact with water, i.e. higher values of $f_{\mathrm{m}}$ and/or $f_{\mathrm{n}}$, resulting in significantly higher value of overall $f$. According to the eqn (3), increasing $f$ value would increase the $\cos \theta_{\mathrm{CB}}$ which in turn decrease $\theta_{\mathrm{CB}}$. Every cycle of hydrophilic treatment and water drop infiltration is expected to progressively increase the amount of collapse, hence increasing $f$ and $\theta_{\mathrm{CB}}$ with each cycle. 


\section{Summary and conclusions}

The surface wettability properties of vertically aligned carbon nanotube carpets on flat graphite substrates have been investigated. These were aligned normal to the surface with some entanglement at the top, much like thick pile of fibrous carpets. As grown pure CNT arrays are seen to be super-hydrophobic, having contact angle about 165 . The surface wettability of these carpets can be altered with short-term microwave oxygen plasma. It is seen that plasma treatment decreases contact angle rapidly with treatment time and by 6 seconds, the carpet becomes super-hydrophilic, with water contact angle below $20{ }^{\circ} \mathrm{C}$ that gets rapidly soaked up by the carpet. This effect can be completely reversed by gentle heating at $110^{\circ} \mathrm{C}$ in air. It is possible to repeat plasma and heat treatment steps multiple times on the same solid to cycle the CNT nano-carpets between hydrophilic and hydrophobic states.

$\mathrm{X}$-ray photoelectron spectroscopy was used to investigate the surface chemical states of the constituent atoms (mostly carbon and added oxygen). It was possible to deconvolute the $\mathrm{C} 1 \mathrm{~s}$ and $\mathrm{O}$ 1s peaks to conclude that the $\mathrm{C}-\mathrm{O} / \mathrm{OH}$ type functional group added by plasma treatment is responsible for hydrophilic behavior of CNT surface and changes in water contact angle was seen to correlate directly with changes in XPS signals related to this functional group.

Simple models for water contact angles on uneven hierarchical surfaces have been discussed. When surface is treated for hydrophilicity, wettability is increased significantly by the presence of nanotubes, as expected from the Wenzel model. For the hydrophobic state, Cassie Baxter model is more appropriate since the water touches only the outer tips of the surface leaving air pockets inside. The inherent contact angle of graphene reported in the literature was used along with microstructural topography analysis to predict the contact angle of vertically aligned carpets. The estimated contact angle is seen to be in very good agreement with experimental values. This model could also be used to explain observed changes in hydrophobichydrophilic cycling seen at spots where the CNT carpet morphology had been altered due to water-drop experiments.

These results offer important insights into tailoring the wettability behavior of carbon nanotube carpets that can heavily impact their applicability in environmental, biomedical and microfluidic devices.

\section{Acknowledgements}

Financial support from the following programs are acknowledged: Ohio Third Frontier Program, NSF-CBET (1449582) and Wright State University PhD. Fellowship. Facilities used were funded by NSF-MRI award and Ohio Board of Regents. The REU component of the NSF-CBET grant enabled undergraduate training in some aspects of this study.

\section{References}

1 W. A. de Heer, Science, 2002, 297, 787.
2 H. Pan, J. Li and Y. P. Feng, Nanoscale Res. Lett., 2010, 5, 654668.

3 E. L. Hopley, S. Salmasi, D. M. Kalaskar and A. M. Seifalian, Biotechnol. Adv., 2014, 32, 1000-1014.

4 K. Balasubramanian and M. Burghard, Anal. Bioanal. Chem., 2006, 385, 452-468.

5 S. M. Mukhopadhyay, A. Karumuri and I. T. Barney, J. Phys. D: Appl. Phys., 2009, 42, 195503.

6 S. M. Mukhopadhyay and A. K. Karumuri, J. Phys. D: Appl. Phys., 2010, 43, 365301.

7 H. Vijwani and S. M. Mukhopadhyay, Appl. Surf. Sci., 2012, 263, 712-721.

8 S. M. M. I. T. Barney, S. Ganguli and A. K. Roy, J. Nanotechnol. Eng. Med., 2012, 3, 31005-31006.

9 E. Maurer, S. Hussain and S. M. Mukhopadhyay, Nanosci. Nanotechnol. Lett., 2011, 3, 110-113.

10 A. Patel, S. Mukundan, W. Wang, A. Karumuri, V. Sant, S. M. Mukhopadhyay and S. Sant, Acta Biomater., 2016, 32, 77-88.

11 E. I. Maurer, K. K. Comfort, S. M. Hussain, J. J. Schlager and S. M. Mukhopadhyay, Sensors, 2012, 12, 8135-8144.

12 A. K. Karumuri, D. P. Oswal, H. A. Hostetler and S. M. Mukhopadhyay, Mater. Lett., 2013, 109, 83-87.

13 A. K. Karumuri, A. A. Maleszewski, D. P. Oswal, H. A. Hostetler and S. M. Mukhopadhyay, J. Nanopart. Res., 2014, 16, 2346.

14 P. C. Ma, N. A. Siddiqui, G. Marom and J. K. Kim, Composites, Part A, 2010, 41, 1345-1367.

15 A. I. Aria and M. Gharib, Langmuir, 2011, 27, 9005-9011.

16 J. I. Paredes, A. Martinez-Alonso and J. M. D. Tascon, J. Mater. Chem., 2000, 10, 1585-1591.

17 T. Young, Philos. Trans. R. Soc. London, 1805, 95, 65-87.

18 R. N. Wenzel, Ind. Eng. Chem., 1936, 28, 988-994.

19 B. D. Cassie, Trans. Faraday Soc., 1944, 40, 546-551.

20 K. Balani, R. G. Batista, D. Lahiri and A. Agarwal, Nanotechnology, 2009, 20, 305707.

21 X. Feng, J. Zhai and L. Jiang, Angew. Chem., Int. Ed. Engl., 2005, 44, 5115-5118.

22 Y. Liu, Z. Lin, W. Lin, K. S. Moon and C. P. Wong, ACS Appl. Mater. Interfaces, 2012, 4, 3959-3964.

23 J. Pan, X. Song, J. Zhang, H. Shen and Q. Xiong, J. Phys. Chem. C, 2011, 115, 22225-22231.

24 A. Chaudhary and H. C. Barshilia, J. Phys. Chem. C, 2011, 115, 18213-18220.

25 L. Li, L. Hua Li, S. Ramakrishnan, X. J. Dai, K. Nicholas, Y. Chen, Z. Chen and X. Liu, J. Phys. Chem. C, 2012, 116, 18334-18339.

26 L. H. Li and Y. Chen, Langmuir, 2010, 26, 5135-5140.

27 C. T. Wirth, S. Hofmann and J. Robertson, Diamond Relat. Mater., 2008, 17, 1518-1524.

28 P. Li, X. Lim, Y. Zhu, T. Yu, C.-K. Ong, Z. Shen, A. T.-S. Wee and C.-H. Sow, J. Phys. Chem. B, 2007, 111, 1672-1678.

29 S. C. Ramos, G. Vasconcelos, E. F. Antunes, A. O. Lobo, V. J. Trava-Airoldi and E. J. Corat, J. Vac. Sci. Technol., B: Microelectron. Nanometer Struct.-Process., Meas., Phenom., 2010, 28, 1153. 
30 S. C. Ramos, G. Vasconcelos, E. F. Antunes, A. O. Lobo, V. J. Trava-Airoldi and E. J. Corat, Diamond Relat. Mater., 2010, 19, 752-755.

31 A. A. Kafi, Z. Chen, X. J. Dai, B. L. Fox, P. R. Lamb and X. Wang, Nanosci. Nanotechnol. Lett., 2013, 4, 344-347.

32 C. H. Chen, H. C. Su, S. C. Chuang, S. J. Yen, Y. C. Chen, Y. T. Lee, T. R. Yew, H. Chen, S. R. Yeh, Y. C. Chang and D. J. Yao, Transducers-Int. Conf. Solid-State Sens., Actuators Microsyst., 15th, 2009, 485501, 967-970.

33 A. K. Karumuri, L. He and S. M. Mukhopadhyay, Appl. Surf. Sci., 2015, 327, 122-130.

34 A. K. Karumuri, D. P. Oswal, H. A. Hostetler and S. M. Mukhopadhyay, Nanotechnology, 2016, 27, 145603.

35 H. Vijwani, PhD Dissertation, Wright State University, 2015.

36 B. T. Quinton, PhD Dissertation, Wright State University, 2016.

37 A. Merenda, E. des Ligneris, K. Sears, T. Chaffraix, K. Magniez, D. Cornu, J. A. Schütz and L. F. Dumée, Sci. Rep., 2016, 6, 31565.

38 M.-S. Chae, J. Kim, D. Jeong, Y. Kim, J. H. Roh, S. M. Lee, Y. Heo, J. Y. Kang, J. H. Lee, D. S. Yoon, T. G. Kim, S. T. Chang and K. S. Hwang, Biosens. Bioelectron., 2016, 4, 31-38.
39 Y. Il Yun, K. S. Kim, S.-J. Uhm, B. B. Khatua, K. Cho, J. K. Kim and C. E. Park, J. Adhes. Sci. Technol., 2004, 18, 1279-1291.

40 E. P. Everaert, H. C. Van Der Mei and H. J. Busscher, J. Adhes. Sci. Technol., 1996, 10, 351-359.

41 N. Chakrapani, B. Wei, A. Carrillo, P. M. Ajayan and R. S. Kane, Proc. Natl. Acad. Sci. U. S. A., 2004, 101, 40094012.

42 C. Py, R. Bastien, J. Bico, B. Roman and A. Boudaoud, Europhys. Lett., 2007, 77, 44005.

43 H. Liu, J. Zhai and L. Jiang, Soft Matter, 2006, 2, 811.

44 J. Liu and G. Gong, IEEE Int. Conf. Nanotechnol., 12th, 2012, 1-4.

45 V. Datsyuk, M. Kalyva, K. Papagelis, J. Parthenios, D. Tasis, A. Siokou, I. Kallitsis and C. Galiotis, Carbon, 2008, 46, 833-840.

46 P. Roach, N. J. Shirtcliffe and M. I. Newton, Soft Matter, 2008, 4, 224.

47 L. He, M. S. thesis, Wright State University, 2016.

48 F. Taherian, V. Marcon, N. F. A. van der Vegt and F. Leroy, Langmuir, 2013, 29, 1457-1465.

49 E. Singh, A. V. Thomas, R. Mukherjee, X. Mi, Y. Peles, Y. Shi and N. Koratkar, ACS Nano, 2013, 7, 3512-3521.

50 C. H. Lee, J. Drelich and Y. K. Yap, Langmuir, 2009, 25, 48534860. 